\title{
Preparation of gold-carbon dots and ratiometric fluorescence cellular imaging
}

Lingyang Zhang, ${ }^{\dagger}$ Donghui Wang, ${ }^{\ddagger}$ Haowen Huang, ${ }^{*}{ }^{\dagger}$ Lanfang Liu, ${ }^{\dagger}$ Yuan Zhou $^{\dagger}$ Xiaodong Xia, ${ }^{\dagger}$ Keqin Deng, ${ }^{\dagger}$ Xuanyong Liu**

${ }^{\dagger}$ Key Laboratory of Theoretical Organic Chemistry and Function Molecule, Ministry of Education, Hunan Provincial University Key Laboratory of QSAR/QSPR, School of Chemistry and Chemical Engineering, Hunan University of Science and Technology, Xiangtan, China.

${ }^{\ddagger}$ State Key Laboratory of High Performance Ceramics and Superfine Microstructure, Shanghai Institute of Ceramics, Chinese Academy of Sciences, Shanghai 200050, China

Corresponding authors

Haowen Huang: hhwn09@163.com, Tel:+86-731-58290045, Fax: +86-731-58290509 Xuanyong Liu: $\quad$ xyliu@mail.sic.ac.cn, Tel:+86-21-52412409, Fax: +86-21-52412409 

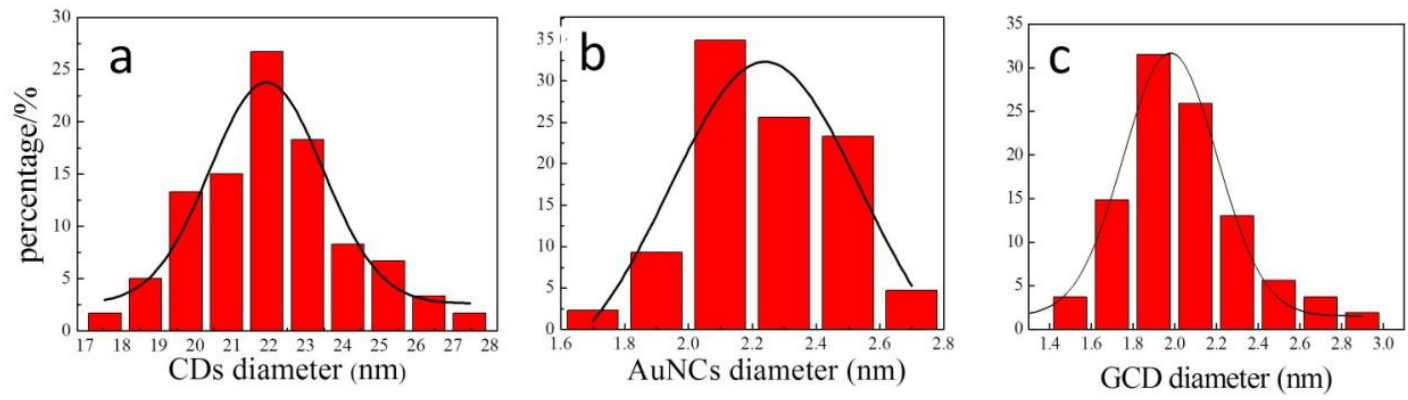

Figure S1. The size distribution of CDs (a), AuNCs (b) and GCDs (c) were prepared by microwave-assisted methods.
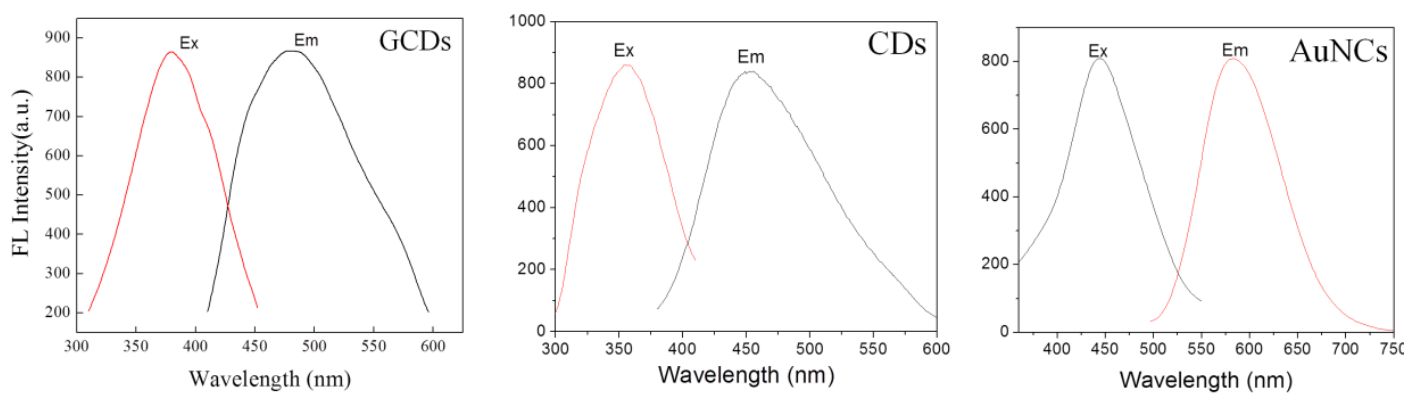

Figure S2. Comparison of fluorescence spectra of GCDs $(\lambda e x=379 \mathrm{~nm}, \lambda \mathrm{em}=480 \mathrm{~nm})$, CDs $(\lambda \mathrm{ex}=354 \mathrm{~nm}, \lambda \mathrm{em}=456 \mathrm{~nm})$ and AuNCs $(\lambda \mathrm{ex}=443 \mathrm{~nm}, \lambda \mathrm{em}=584 \mathrm{~nm})$.

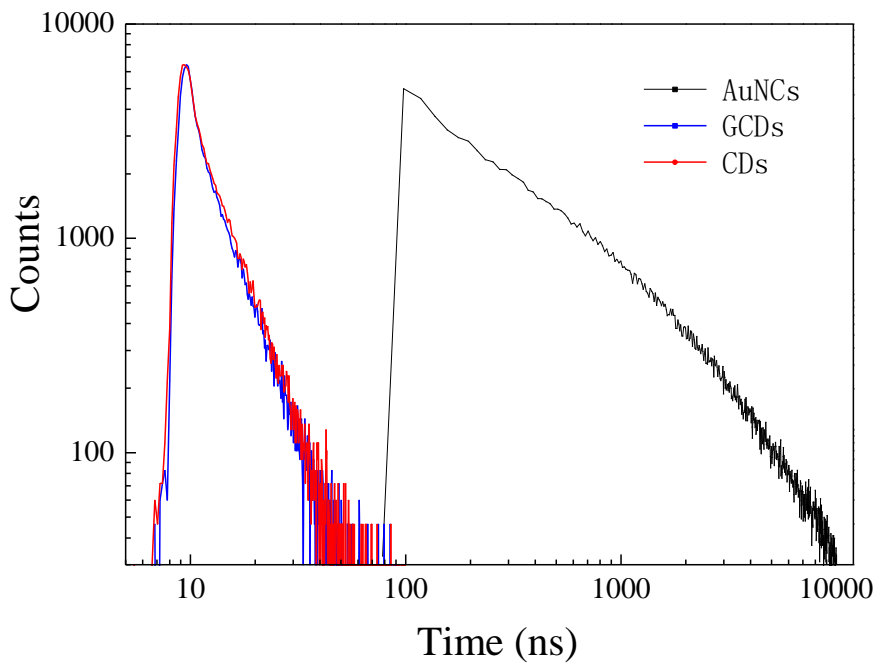

Figure S3. Fluorescence decays acquired from GCDs $\left(\tau_{1}=0.8091 \mathrm{~ns}(46.32 \%)\right.$, $\left.\tau_{2}=4.0006 \mathrm{~ns}(53.68 \%)\right)$, CDs $\left(\tau_{1}=0.7773 \mathrm{~ns}(38.21 \%), \tau_{2}=4.0087 \mathrm{~ns}(61.79 \%)\right)$ and AuNCs $\left(\tau_{1}=285.0267 \mathrm{~ns}(27.98 \%), \tau_{2}=1863.2993 \mathrm{~ns}(72.02 \%)\right)$. 


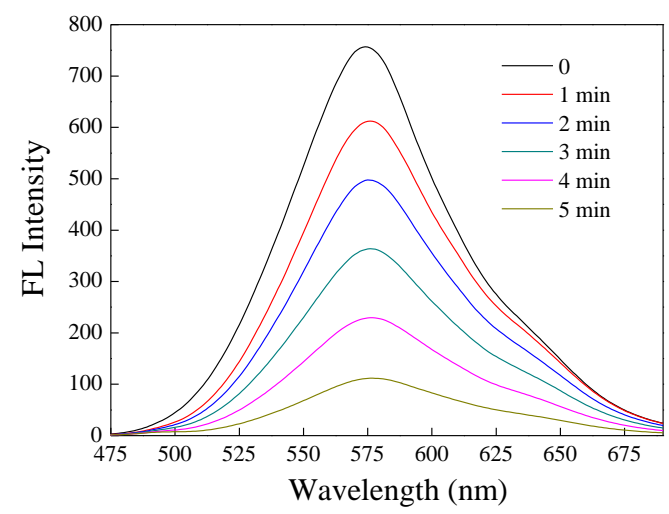

Figure S4. Gradually decreasing fluorescence after addition of $\mathrm{Na}_{2} \mathrm{~S}_{2} \mathrm{O}_{3}$ into AuNCs at different time intervals
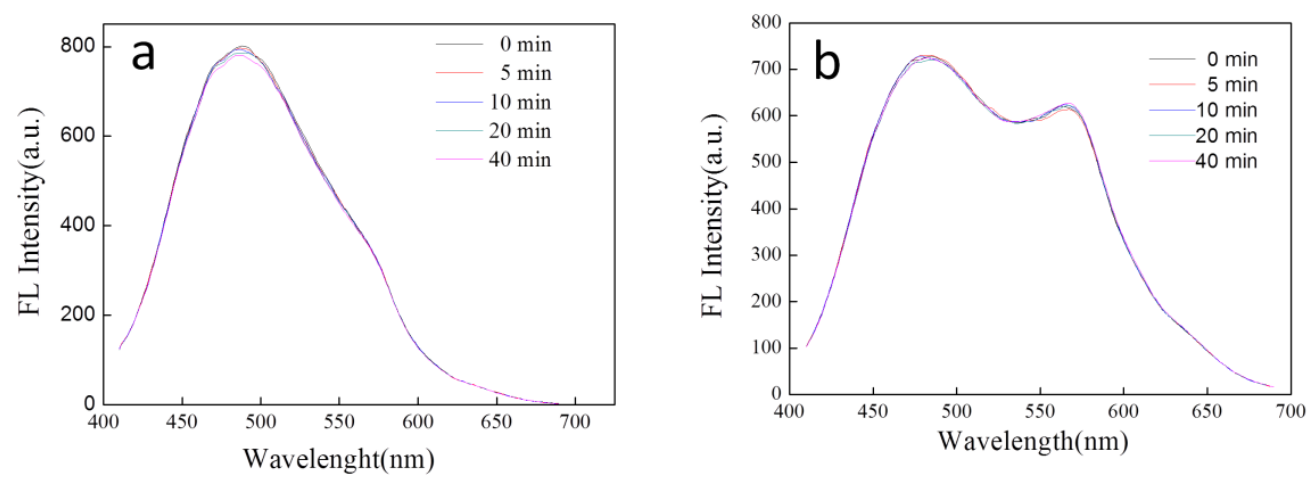

Figure S5. Variation of fluorescent intensity of GCDs under successive UV light irradiation at time intervals, $a$ represents the as-prepared GCDs, $b$ is the dual-emission after the as-prepared GCDs reacting with GSH.

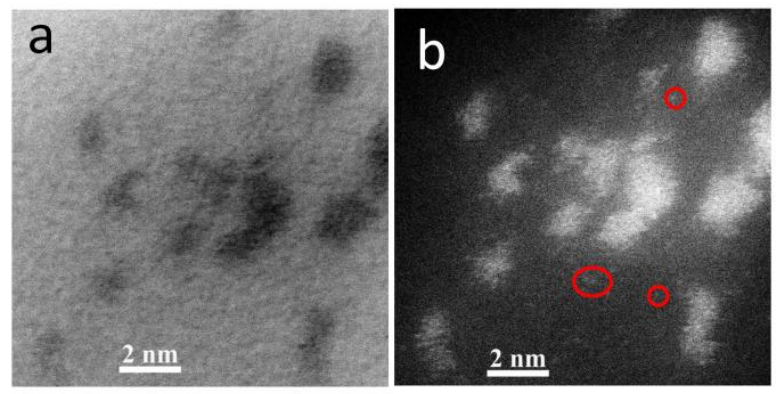

Figure S6. High resolution STEM bright field image (a) and HAADF-STEM (b) of GCDs. In the circles of HAADF-STEM image, every light spot represents one $\mathrm{Au}$ atom in the circles, which illustrates the $\mathrm{Au}$ nanoclusters consisting of several $\mathrm{Au}$ atoms. 


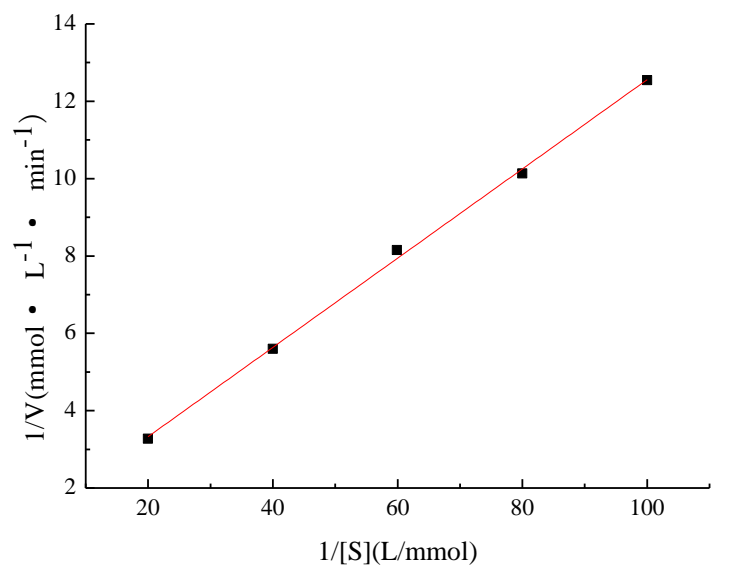

Figure S7. The standard curve of the assay detecting enzymatic parameters of GCDs. $\mathrm{Y}=0.11539 \mathrm{X}+1.01967, \mathrm{~K}_{\mathrm{m}}=113.08 \mathrm{mmol} / \mathrm{L}, \mathrm{V}_{\max }=0.9807 \mathrm{mmol} \cdot \mathrm{L}^{-1} \cdot \mathrm{min}^{-1}, \mathrm{R}^{2}=0.9983$.

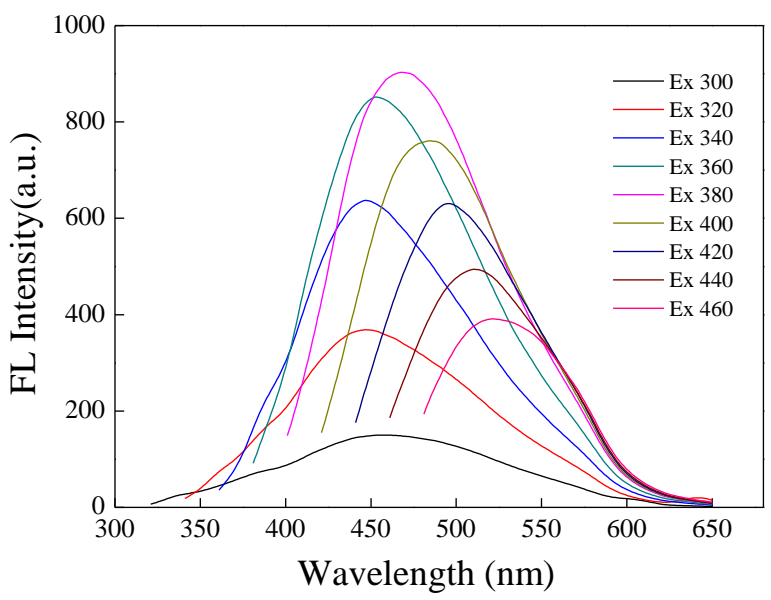

Figure S8. Emission spectra of GCDs after etched by aqua regia as a function of excitation wavelength. 


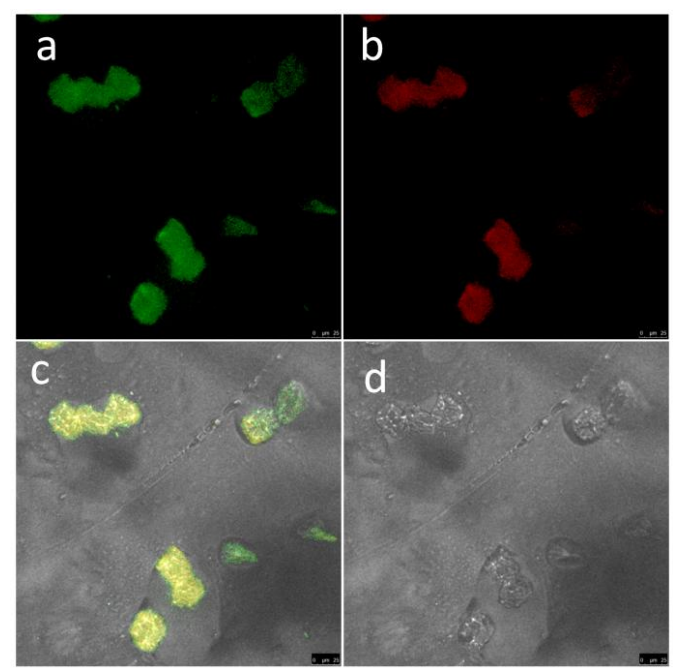

Figure S9. Confocal microscopic images of normal MC3T3-E1 cells incubated with GCDs for $10 \mathrm{~h}$ : (a) dark-field by 420-480 $\mathrm{nm}$ blue channel, (b) dark-field by 550-630 nm red channel, (c) overlay images and (d) bright-field of the MCF-7 cells.

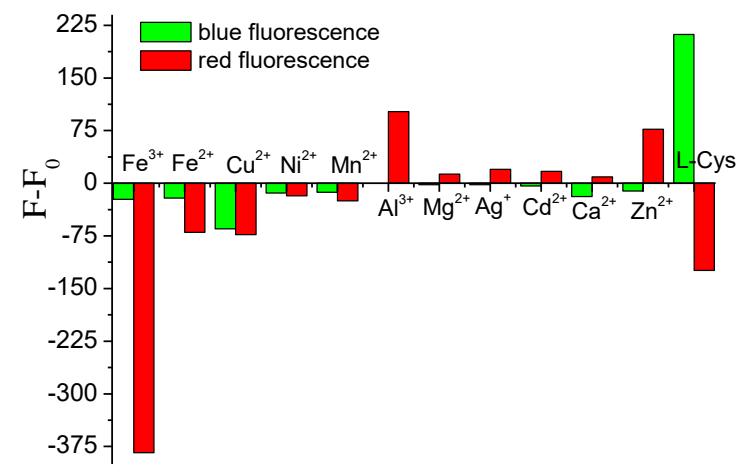

Figure S10. Comparison of CDs, AuNCs and GCDs fluorescent response to the same target species.

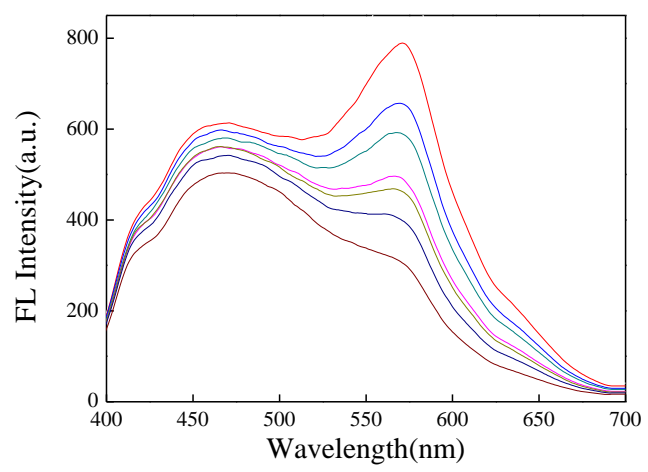

Figure S11. Red fluorescence quenching of GCDs with increasing $\mathrm{Fe}^{3+}$ concentration (from top to bottom: $0,1 \times 10^{-6}, 3 \times 10^{-6}, 4 \times 10^{-6}, 5 \times 10^{-6}, 6 \times 10^{-6}, 7 \times 10^{-6} \mathrm{M}$ ). 